\title{
Lenguaje y contenido audiovisual de los programas en Internet frente a los programas de televisión convencional*
}

\author{
Jorge Eduardo Urrea Giraldo**
}

\section{Resumen}

Introducción. Los nuevos soportes de exhibición para material de imagen en movimiento han planteado la pertinencia de identificar si hay modificaciones en el lenguaje audiovisual según las características del soporte de emisión. Al comparar piezas audiovisuales emitidas en soportes convencionales y convergentes en la actualidad, se aprecia el uso de elementos de la narrativa web, modificación y uso selectivo de los elementos del lenguaje audiovisual. Objetivo. Comparar el lenguaje y contenido audiovisual de programas en Internet frente a la televisión convencional. Materiales y métodos. Se constituyó una muestra intencional de 17 programas, distribuidos en tres canales de televisión convencional con sus respectivos portales de Internet, y un blog de producción audiovisual exclusiva para la web. Por medio de una plantilla de observación elaborada en Excel y entrevistas semiestructuradas dirigidas a los emisores, se analizaron las categorías de lenguaje y contenido audiovisual. Resultados. Fueron pocas las diferencias encontradas. En los programas exclusivos para la web se aprecia: fragmentación de contenidos, corta duración de las piezas, preponderancia de planos medios y cerrados y montaje lineal, ágil y rítmico, además de mayor nivel de interactividad. Los contenidos ofrecidos por demanda corresponden mayoritariamente a programas exclusivos para la web; mientras los programas emitidos en señal convencional, también disponen de señal de flujo o streaming en los portales web. Conclusiones. Las producciones carecen de un lenguaje audiovisual propio para los programas exhibidos en la web, las modificaciones se identifican en las narrativas que incorporan elementos gráficos determinados por las tecnologías de la información y la comunicación.
Palabras clave: lenguaje audiovisual, contenidos, televisión, internet, TIC

\section{Language and audiovisual content of programs on Internet, compared to those from conventional TV}

\begin{abstract}
Introduction. The new exhibition ways for the inmotion images have brought the necessity to identify if there are modifications in the audiovisual language according to the characteristics of the media. When audiovisual works are broadcasted on conventional media and on those that are converging nowadays, the use of elements from the web's narrative can be appreciated, along with modifications and a selective use of the audiovisual language's elements. Objective. Compare the language and the audiovisual contents from Internet and conventional TV programs. Materials and methods. An intentional sample of 17 programs distributed in three conventional TV channels with their respective Internet sites and an exclusive audiovisual production blog for the web, were used. By the use of an observation template, made in Excel, and semi-structured interviews to the transmitters, the language and audiovisual content categories were analyzed. Results. The differences found were not many. In the programs made exclusively for the web, a fragmentation of the contents, a short duration of the programs, a preponderance of medium shots and close-ups and a linear, agile and rhythmic edition can be seen, plus a higher interactivity level. On-demand contents are mostly for programs that are exclusively for the web, while the programs broadcasted on the conventional TV signal also have streaming when they are available online. Conclusions. The productions do
\end{abstract}

\footnotetext{
* Artículo derivado del trabajo de maestría "Estudio comparativo de las características de los contenidos y el lenguaje audiovisual usados en programas de televisión convencional y programas en internet", realizado en el II semestre académico de 2012 con el aval de la Universidad Pontificia Bolivariana, sede Medellín.

** Comunicador Social y Periodista, Magister en Comunicación Digital. Profesor de Planta programa de Comunicación Audiovisual y Multimedios Fundación Universitaria del Área Andina - Pereira.
} 
not have an individual language for the programs intended for online exhibition. The modifications are identified in the narratives that incorporate graphic elements determined by the information and communication technologies.

Key words: audiovisual language, contents, television, internet, IT.

\section{Linguagem e conteúdo audiovisual dos programas em internet frente aos programas de televisão convencional}

\section{Resumo}

Introdução. Os novos suportes de exibição para material de imagem em movimento propuseram a pertinência de identificar se há modificações na linguagem audiovisual segundo as características do suporte de emissão. Ao comparar peças audiovisuais emitidas em suportes convencionais e convergentes na atualidade, aprecia-se o uso de elementos da narrativa web, modificação e uso seletivo dos elementos da linguagem audiovisual. Objetivo. Comparar a linguagem e conteúdo audiovisual de programas em Internet frente à televisão convencio- nal. Materiais e métodos. Constituiu-se uma mostra intencional de 17 programas, distribuídos em três canais de televisão convencional com seus respectivos portais de Internet, e um blog de produção audiovisual exclusiva para a web. Por meio de uma planilha de observação elaborada em Excel e entrevistas semiestruturadas dirigidas aos emissores, analisaram-se as categorias de linguagem e conteúdo audiovisual. Resultados. Foram poucas as diferenças encontradas. Nos programas exclusivos para a web se aprecia: fragmentação de conteúdos, curta duração das peças, preponderância de planos meios e fechados e montagem linear, ágil e rítmico, além de maior nível de interatividade. Os conteúdos oferecidos por demanda correspondem maioritariamente a programas exclusivos para a web; enquanto os programas emitidos em sinal convencional, também dispõem de sinal de fluxo ou streaming nos portais web. Conclusões. As produções carecem de uma linguagem audiovisual próprio para os programas exibidos na web, as modificações se identificam nas narrativas que incorporam elementos gráficos determinados pelas tecnologias da informação e a comunicação.

Palavras importantes: linguagem audiovisual, conteúdos, televisão, internet, TIC.

\section{Introducción}

El área de las comunicaciones enfrenta novedosos y acelerados desarrollos tecnológicos, como la incursión de las Tecnologías de la Información y la Comunicación-TIC que fusionan sistemas para transmisión de contenidos, lo que contrasta con una aplicación pertinente de medios alternativos, y la necesidad de generar cambios enfocados a los requerimientos de la producción para la web. El estudio del lenguaje audiovisual se basó en los planteamientos de Eco (1999), Durand (2000) y Barthes (1991), quienes lo entienden como la interconexión de signos visuales y sonoros, lo que implica diversidad de formas para representar la realidad. Se analizó la configuración de los elementos morfológicos y sintácticos de la imagen desde la perspectiva propuesta por Siety (2004), Palazón (1998) y Martin (1990); así se puede identificar estructuralmente la forma como se construye el mensaje audiovisual. Desde la óptica de Finkel (2009), Castells (2001) Salaverria (2005) y Scolari (2013) se definen los elementos que determinan la convergencia y su uso en las narrativas audiovisuales según las recomendaciones técnicas planteadas por Bourne \& Burstein (2009) en las plataformas descritas por Harte, McGarrahan, \& Chandler (2011).

A pesar del interés de los canales de televisión por ofrecer contenidos audiovisuales en Internet, y del reconocimiento de estos productos por los consumidores en la web, es necesario acudir a modelos productivos que generen contenidos específicos para programas en Internet con lenguaje y procedimientos determinados. Así lo plantean Bourne \& Burstein (2009) al afirmar que se debe tener en cuenta la compresión por la pérdida de detalles en pequeñas pantallas, lo que exige mayor uso de primeros planos; asimismo la edición se debe caracterizar por incluir más cortes y menos efectos.

Identificar coincidencias y discrepancias de los productos audiovisuales expuestos en Internet y televisión es un insumo valioso para la indus- 
tria audiovisual formal, y para los realizadores independientes y aficionados, que requieren conocer las características que exigen los nuevos medios, como lo recomienda Manovich (2005).

\section{Materiales y métodos}

A través de la metodología de investigación cualitativa con enfoque descriptivo, mediante la técnica de análisis de contenido, se realizó el registro, análisis e interpretación del lenguaje audiovisual y los contenidos de diecisiete programas transmitidos en televisión convencional e Internet. Estos fueron seleccionados de manera deliberada con fines comparativos, sobre la base de criterios que corresponden a las categorías de lenguaje audiovisual, contenidos y narrativas audiovisuales. La muestra se conformó con una emisión por programa, tomadas de los tres canales de televisión convencional: Canal Caracol y Señal Colombia, de radiodifusión nacional, y el canal regional Telecafé, cada uno con los portales de Internet respectivos; se añadió un elemento innovador que fue un blog de producción nacional por medio del que se transmite el programa "Susana y Elvira La serie".

La selección se realizó bajo el criterio de representación de los principales servicios de televisión en Colombia, según su clasificación de cubrimiento y carácter organizacional. De cada canal de televisión y portal web, se tomó una muestra de la programación de acuerdo con los siguientes criterios: a) Mínimo tres programas por cada canal televisivo, distribuidos en las franjas: mañana, tarde y noche, b) Disponibilidad de programas con emisión en simultánea a través de flujo (streaming) y/o vídeo por demanda (VoD) según la información publicada en las páginas web respectivas, c) Reconocimiento de los programas en cada medio según la información publicada en sus páginas web, d) Programas exclusivos para la web, coincidentes con el periodo de tiempo destinado al trabajo de campo realizado, e) Un programa exhibido en plataforma convergente creada en Colombia con producción de contenidos originales y creativos para la web; en este caso particular se seleccionó el blog "Susana y Elvira La serie" por producir una serie Web de ficción. El trabajo de campo inició con la aplicación de las entrevistas semiestructuradas, dirigidas a los representantes legales de los canales seleccionados en la muestra. Para el análisis de los programas se observó la programación de la muestra de manera simultánea en televisión e Internet; en el primer medio se utilizó un televisor común marca LG de 14 pulgadas con tecnología de Tubo de rayos catódicos (CRT), conectado a un servicio de televisión por cable; se accedió a Internet con un computador portátil MacBook Pro conectado vía WiFi a banda ancha de una mega. La información fue diligenciada en la ficha de observación diseñada para el análisis de contenido, la cual fue aplicada en ambos medios de manera simultánea.

\section{Resultados}

La comparación del lenguaje audiovisual utilizado en los canales de emisión que componen la muestra arroja como resultado la poca diferencia entre el lenguaje audiovisual usado en los programas de televisión convencional e Internet. La principal diferencia se aprecia en los programas exclusivos para la web; en su mayoría corresponden al género informativo y al formato magacín, con excepción de la serie de ficción "Susana y Elvira la serie".

La mayoría de programas de la muestra evidencian corta duración de las piezas con un promedio de seis minutos, montaje lineal, ágil y rítmico, movimientos de cámara moderados y una ponderada primacía en la escala de planos, tendente a encuadres ajustados o cerrados; los programas analizados encajan en una morfología con elementos visuales figurativos, aunque la lectura de la imagen depende del bagaje del receptor; algunos elementos denotativos simples. En lo sonoro hay privilegio de la palabra como eje conductor en las historias y de la música incidental como acompañamiento en la mayoría de oportunidades. En estos casos la música cumple un papel narrativo, o de refuerzo de la intención expresiva en pocas oportunidades, en particular sucede en la serie de ficción. En cada medio se encuentran factores a destacar, que aportan a la construcción de nuevas formas de narrar en el paso de lo convencional a lo convergente; se reconocen falencias relacionadas con el tamaño de planos, movimientos de cámara, efectos de pos- 
producción, entre otros, que son evidentes en el estado de la producción de las piezas que se exhiben en la web.

\section{Caracol Televisión}

De acuerdo con la muestra, Caracol Tv es el canal con mayor cantidad de productos disponibles para la web, tiene más cercanía a la producción de piezas vistas en otras pantallas, porque se reconocen usos y costumbres de los usuarios de las TIC, que permiten la no linealidad presentada en los contenidos ofrecidos bajo la modalidad VoD; se pasa del "prime time" al "my time" como afirma Andrew Green (2010) cuando plantea que las personas cada día controlan más su entorno; lo importante es el manejo que cada uno da al tiempo, y no cómo lo programan los canales convencionales.

Se destaca la fragmentación de contenidos para facilitar el consumo en la red, de manera que no es necesario ver la totalidad de la pieza audiovisual, ya que se puede acceder fácilmente a la sección que corresponde a la modalidad VoD; estas prácticas de fragmentación son comunes en los informativos donde se ponen a disposición las notas de manera independiente. El entorno web dispuesto para la presentación de contenidos de flujo y los dispuestos para consumo bajo demanda son adecuados en términos de tamaño de la ventana de visualización y accesibilidad de elementos web como interacción, personalización y multimedialidad. Sin embargo, la calidad de compresión es muy baja, lo que degrada la definición de la imagen y genera pixeles por compresión evidente en los movimientos de cámara y en los movimientos internos del plano.

\section{Señal Colombia}

Por la calidad final de las piezas, representada en el uso del lenguaje audiovisual de manera pertinente para la intención narrativa, se destaca la correcta configuración técnica y los contenidos estructurados y fundamentados. En todos los programas de la muestra correspondiente a Señal Colombia sobresale la estructura modular, que permite la fragmentación en la edición de piezas cortas con sentido completo para ser vistas en la red; sin embargo, no se identificaron productos para consumir bajo la modalidad VoD.

Al contrastar la programación ofrecida bajo la modalidad streaming no corresponde con la totalidad de la emitida en la señal de aire; es decir, hay tiempos en la plataforma en que no es posible observar el canal.

El entorno web de Señal Colombia está organizado de manera que es fácil encontrar la programación, porque está catalogada de acuerdo con los géneros; sin embargo, en la señal de flujo se evidencia una pantalla muy pequeña, lo que hace difícil ver los contenidos. Para enmendar ese inconveniente está habilitada la opción de pantalla completa que despliega una imagen más grande; esta, a su vez, es más defectuosa en términos de definición. Además de los evidentes pixeles por compresión, en la opción de pantalla completa se elimina totalmente la posibilidad de acceder a información adicional, ejercer interactividad o acceder a contenidos multimediales, al tiempo que se observa la señal de flujo.

Cada programa cuenta con un minisitio en el que es posible acceder a información general y múltiples piezas multimediales, entre ellas, audios con la banda sonora de los programas, clips promocionales y fotografías. Las opciones de interactividad en redes sociales no están ligadas a la emisión de los programas, de manera que se pierde la opción de compartir en tiempo real y comentar los contenidos que se están emitiendo desde una misma interfaz.

\section{Telecafé}

Entre los ocho canales regionales colombianos, Telecafé es pionero en la producción de programas exclusivos para la web con el magacín Punto Tv, y ha invertido recursos de producción en la exploración de formas narrativas para este programa, representadas en elementos gráficos, movimientos de cámara y puntos de vista (POV). Telecafé pone a disposición de los usuarios de la web, el noticiero Telecafé noticias y un documental cultural, bajo la modalidad VoD. En la entrevista, el canal manifestó producir contenidos especiales para transmitir vía streaming, y coincidió en la importancia de 
este aspecto, debido a la cantidad de productos que ofrece.

Las respuestas que corresponden al eje de formatos y modelos de producción para web revelan poco conocimiento y aplicación de las narrativas digitales, ya que se basan en los formatos y conceptos existentes de la televisión convencional para condicionar las piezas de programas producidos para Internet. Se infiere que no se han desarrollado propuestas basadas en las características del nuevo medio, ni se genera un lenguaje audiovisual consciente para dichas plataformas.

En lo concerniente a la interactividad, declara hacerlo mediante redes sociales y blogs, pero en confrontación con los programas analizados solo se encontró el uso de redes sociales en el contenedor de la mañana, "Buenos días, eje". No se evidencian intenciones de explorar otras formas de interacción ni de narrativas rizomáticas; sin embargo, en la entrevista se evidencia la existencia de protocolos de edición para la configuración técnica, que busca la calidad de las piezas puestas en la red.

El entorno web del canal es de fácil acceso, y la calidad de compresión es aceptable, pero la interfaz no cuenta con un diseño complementario que permita desarrollar las posibilidades de las narrativas web, de tal manera que la interactividad, la personalización y la multimedialidad son escasas.

\section{Susana y Elvira La serie}

Se destaca por el género y formato elegidos para emitir en la plataforma convergente sobre el formato web Blog. Es una serie web de ficción que según la información obtenida en la entrevista, "emula el espíritu del blog"; porque se aprecian diferencias en las formas narrativas, desde las estructuras del guion y los usos del lenguaje audiovisual, por ejemplo, la interrupción "natural de la escena" que hacen los protagonistas para dirigirse a los usuarios de la red y el acompañamiento de elementos gráficos que emulan los entornos virtuales. Se identifica en la intencionalidad del lenguaje audiovisual aspectos como la ausencia de tiempos muertos en el montaje o minimización de los movimientos de cámara, justificados sólo en las acciones de los actores.

En la entrevista quedó claro que la selección de la plataforma pretende ser coherente con el origen de los contenidos, ya que los argumentos están basados en el blog del mismo nombre. Hay claridad en las posibilidades y limitaciones que ofrece la plataforma seleccionada para la exhibición y tienen reconocidos los dispositivos en que los usuarios consumen los contenidos de la serie, y hacen medición del impacto a través de herramientas web, como google analytics y las estadísticas de las plataformas donde están alojados los capítulos.

La promoción de la serie está basada primordialmente en free press y campañas virales en redes sociales; se identifica un uso adecuado de la interfaz con una pantalla donde se puede apreciar cómodamente la serie y ejercer interactividad al mismo tiempo, al dejar mensajes en las redes sociales y en el foro; también se aprecian elementos multimediales como la banda sonora. La interfaz facilita el acceso a todos los capítulos de la serie que pueden ser encontrados fácilmente en la misma página.

\section{Conclusiones}

En relación con el lenguaje y los contenidos audiovisuales se concluye que la teoría alrededor del lenguaje audiovisual conserva los fundamentos establecidos coetáneamente con la aparición del cine sonoro, desarrollado por estudiosos del séptimo arte como Marcel Martin (1990) y Alfonso Palazón (1998). Dichas bases se aplican de manera no tan rigurosa, porque es frecuente que los directores exploren con estas en los medios audiovisuales convencionales. Las investigaciones actuales se desarrollan, en su mayoría, alrededor de narrativas y de la forma como la producción de contenidos para los programas de Internet y Cross Media han modificado las lógicas de producción, de manera que los modelos han debido incluir nuevos procesos y elementos de la web.

Se identifican modificaciones al lenguaje audiovisual que no implican cambiar los elementos que determinan la morfología y la sintaxis, sino complementarlos con narrativas propias 
de los medios digitales. En la actualidad dicha complementariedad solo se aprecia en algunos programas de la oferta televisiva y fundamentalmente consiste en interactividad limitada a redes sociales y foros, además de elementos multimedia en los portales web. Se constató que una de las modificaciones más significativas consiste en la fragmentación de los contenidos para ser consumidos en piezas más cortas y con sentido completo; la plataforma tradicional privada es la que más realiza este tipo de procedimientos a sus contenidos, particularmente en los informativos y programas de entretenimiento.

No se evidencia la creación de un lenguaje audiovisual propio para los programas exhibidos en la web, aunque con la puesta al aire de contenidos con imagen en movimiento, se ha visto modificado el lenguaje existente, al asumir características de las narrativas web con la creación de roles y dinámicas de producción como la inclusión de elementos gráficos determinados por las TIC. Sin embargo, el lenguaje audiovisual usado en los programas de Internet es el mismo de los transmitidos en televisión convencional, pero con restricciones fijadas por las características narrativas, en las que se requieren inmediatez e interactividad, además de las características técnicas del nuevo medio, como tamaño de las pantallas y calidad de compresión de vídeo, necesaria para garantizar la transferencia de audio y vídeo de manera fluida.

Las formas de exhibición se encuentran en continua modificación*, pues se publican obras bajo la modalidad VoD que ya han sido transmitidas en plataformas convencionales y recobran vigencia en la red, como el caso de telenovelas y seriados producidos sobre los cánones del modelo productivo audiovisual convencional.

Se considera que en la actualidad el lenguaje audiovisual cumple las mismas funciones en ambas plataformas, pues en definitiva busca comunicar mediante la puesta en común de elementos de audio e imagen. Al estudiar las características que los medios convencionales han tomado "prestadas" de la web, como info- gráficos, multimedia, interactividad, etc., aplicados a las piezas audiovisuales puestas en línea, se encuentra que estas podrían encajar en los componentes morfológicos del lenguaje audiovisual clásico, como aquellos elementos visuales representados en gráficos, textos, fotografías, entre otros, y elementos sonoros como voz, música y efectos especiales, entre otros.

Rodríguez (1998) indica que el lenguaje en cualquiera de las dos plataformas aplica los códigos narrativos con el mismo objetivo de solucionar los problemas de interpretación que surgen de las diferencias entre la percepción directa de la realidad y la que representan las formas audiovisuales. Las diferencias halladas entre las narrativas de las plataformas convencionales y las convergentes se determinan por las características técnicas de cada medio y está dada por la puesta en común de las posibilidades narrativas que tienen los soportes utilizados para la emisión, el espectro electromagnético y el ciberespacio.

En síntesis, se concluye que hay una conciencia incipiente sobre la importancia de adaptar el lenguaje y los contenidos audiovisuales a las condiciones de la web, en las pocas piezas realizadas para la convergencia y las intenciones manifiestas en las entrevistas diligenciadas por los representantes de ambas plataformas-excepto Canal Caracol-. Se hace evidente la necesidad de encontrar nuevas formas de narrar, adaptadas a Internet y al vertiginoso crecimiento del uso de dispositivos para el consumo de imagen en movimiento, diferentes al aparato de televisión, además de establecer contacto directo en tiempo real con los televidentes, independiente de la forma de difusión y exhibición.

\section{Referencias bibliográficas}

- Barthes, R. (1991). El imperio de los signos. Madrid: Mondadori.

- Bourne, J., \& Burstein, D. (2009). Web video: Making it great, getting it noticed. San Francisco EE. UU.: Peachpit Press.

Ello no es relativo al lenguaje audiovisual sino a las dinámicas de la industria. 
- Castells, M. (2001). Internet y la sociedad en red. Persona y sociedad XV (2).

- Durand, G. (2000). La imaginación simbólica. Buenos Aires: Amorrortu.

- Eco, U. (1999). La estrategia de la ilusión. Barcelona: Lumen.

- Finkel, M. (2009). Convergencia digital. Guatemala: Universidad de San Carlos.

- Green, A. (2010). From prime time to my time. Londres: Warc.

- Harte, L., McGarrahan, R., \& Chandler, M. (2011). How to set up and run Internet TV systems. Fuquay Varina: Althos Publishing.

- Manovich, L. (2005). El lenguaje de los nuevos medios de comunicación. Barcelona: Paidós Ibérica.
- Martín, M. (1990). El lenguaje del cine. Barcelona: Gedisa.

- Palazón, A. (1998). Lenguaje audiovisual. Madrid: Acento.

- Rodríguez, Á. (1998). La dimensión sonora del lenguaje audiovisual. Barcelona: Paidós.

- Salaverría, R. (2005). Cibermedios, Impacto de internet en los medios de comunicación en España. Sevilla: Comunicación Social Ediciones y Publicaciones.

- Scolari, C. (2013). Narrativas Transmedia. Barcelona: Deusto Editores.

- Siety, E. (2004). El plano en el origen del cine. Barcelona: Paidós. 